\title{
On the Second-Order Shape Derivative of the Kohn-Vogelius Objective Functional Using the Velocity Method
}

\author{
Jerico B. Bacani and Julius Fergy T. Rabago \\ Department of Mathematics and Computer Science, College of Science, University of the Philippines Baguio, Governor Pack Road, \\ 2600 Baguio City, Philippines
}

Correspondence should be addressed to Jerico B. Bacani; jicderivative@yahoo.com

Received 31 July 2015; Revised 11 November 2015; Accepted 11 November 2015

Academic Editor: Julio D. Rossi

Copyright (C) 2015 J. B. Bacani and J. F. T. Rabago. This is an open access article distributed under the Creative Commons Attribution License, which permits unrestricted use, distribution, and reproduction in any medium, provided the original work is properly cited.

\begin{abstract}
The exterior Bernoulli free boundary problem was studied via shape optimization technique. The problem was reformulated into the minimization of the so-called Kohn-Vogelius objective functional, where two state variables involved satisfy two boundary value problems, separately. The paper focused on solving the second-order shape derivative of the objective functional using the velocity method with nonautonomous velocity fields. This work confirms the classical results of Delfour and Zolésio in relating shape derivatives of functionals using velocity method and perturbation of identity technique.
\end{abstract}

\section{Introduction}

Shape optimization is a key research topic with many applications in various fields of pure and applied sciences, especially in biomechanics and engineering (cf. [1,2] for applications in structural mechanics, [3] for some applications in fluid mechanics or aerodynamics, and [4] for other applications). A typical problem in this line of research is to find a domain, for instance, $\Omega$, in a set of admissible domains $\mathscr{A}$ such that an objective functional $J$ achieves a minimum (or maximum) on it [3]. For instance, suppose, among all three-dimensional shapes of given volume, that we wish to find the one which has a minimal surface area. In this particular case, the problem can be described mathematically as finding the minimum of $J(\Omega)=\operatorname{Area}(\partial \Omega)$ with the constraint $E(\Omega)=\operatorname{Volume}(\Omega)-$ constant. Obviously, the answer to this question would be the sphere. In general and in most cases of greater interest, shape optimization problems can be described mathematically as

$$
\begin{array}{ll}
\min _{(u, \Omega)} & J(u, \Omega) \\
\text { s.t. } & E(u, \Omega)=0, \quad \Omega \in \mathscr{A},
\end{array}
$$

where the state $u$ is the solution to a partial differential equation (PDE) $E$ on the domain $\Omega$. For an extensive introduction to shape optimization problems, we refer to the book of Delfour and Zolésio [5] (see also [6]).

Recently, there has been an increasing interest in the applications of shape optimization in the study of Bernoulli problems. Abda et al. [7] rephrased the Bernoulli problem into a shape optimization problem and explicitly determined the shape derivative of the cost functional being studied. In [8], a framework for calculating the shape Hessian for the domain optimization problem with a PDE as the constraint was presented. In [9], a similar approach as in [8] was applied in solving a shape optimization problem.

Another way to approach the solutions of shape optimization problems is through iterative methods. For the past few decades, several numerical methods have been developed to solve the two-dimensional Bernoulli problem (see, e.g., [10-13]). These strategies were also developed based on reformulating the Bernoulli problem as a shape optimization problem. This reformulation can be achieved in several ways. For instance, for a given domain, one can choose one of the boundary conditions on the free boundary to obtain a well-posed state equation. The domain is determined by the requirement that the other condition on the free boundary is satisfied in a least square sense (see [13-15]). 
Many authors have also studied the second variation of a cost functional for linear PDEs. Building on the shape optimization setting that is based on the perturbation of the identity method introduced by Murat and Simon (cf. $[16,17]$ ), Fujii [18] used a second-order perturbation of the identity along the normal of the boundary for second-order elliptic problems in 1986. Simon [19] computed the second variation via the first-order perturbation of the identity in 1988. A general approach via the velocity method (Figure 2) was systematically characterized by Delfour and Zolésio [20, 21], and they computed the shape Hessian for a simple Neumann problem in [20] and a nonhomogeneous Dirichlet problem in [21].

However, a standard approach in dealing with the solution to (1) requires some information on gradients. So shape derivatives are essential in understanding the problem.

The recent paper focuses on the exterior Bernoulli free boundary problem (FBP). As far as the authors are concerned, the same functional was first studied by Eppler and Harbrecht and published in [22] wherein the first-order shape derivative, or equivalently the shape gradient, was derived for arbitrary variations in terms of the perturbation of the identity. Moreover, the second-order shape derivative, or equivalently the shape Hessian, has been computed and analyzed for the special cases of star-like domains. As a main result, by analyzing the shape Hessian at the optimal domain, Eppler and Harbrecht found out that the optimization problem is algebraically ill posed. In the present paper, the same functional is studied again but we focus on the application of velocity method in dealing with shape optimization problem. It would be a challenging research in the near future to study the ill-posedness of the shape optimization problem for general domains, as well as the comparison of the shape Hessians in this paper from [22] for the former uses Cartesian coordinates, while the latter used spherical/polar coordinates. The nice thing in the present paper is that the results attest to classical results in shape optimization problems.

Now, the exterior Bernoulli FBP is formulated as follows.

Given a bounded and connected domain $A \subset \mathbb{R}^{2}$ with a fixed boundary $\partial A:=\Gamma$, we need to find a bounded connected domain $B$ with a free boundary $\Sigma$ that contains the closure of $A, \bar{A}$, and an associated real-valued (state) function $u$ defined on $\Omega$ (where $\Omega$ is the annulus formed by $B$ and $A$; refer to Figure 1) such that both unknowns $u$ and $\Omega$ satisfy the following boundary value problem:

$$
\begin{aligned}
-\Delta u=0 & \text { in } \Omega, \\
u=0 & \text { on } \Sigma, \\
u=1 & \text { on } \Gamma, \\
\frac{\partial u}{\partial \mathbf{n}}=\lambda & \text { on } \Sigma,
\end{aligned}
$$

where $\lambda<0$.

In recent papers, Bacani and Peichl employed shape optimization methods to study the exterior Bernoulli FBP by

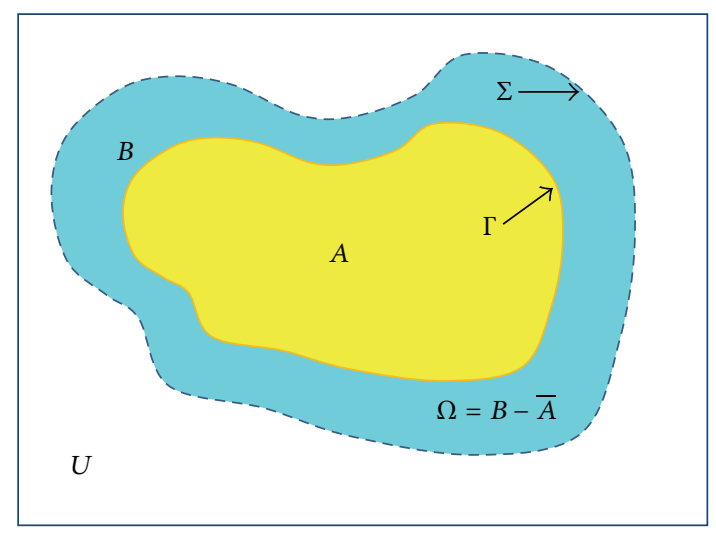

FIGURE 1: The exterior Bernoulli free boundary problem.

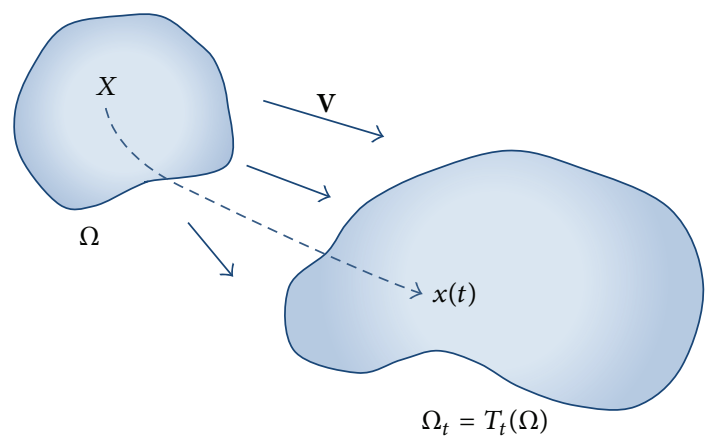

FIgURE 2: Transport of $\Omega$ by velocity method.

reformulating it into Kohn-Vogelius-type cost functional $J_{\mathrm{KV}}$, which is defined as

$$
J(\Omega):=J_{\mathrm{KV}}=\frac{1}{2} \int_{\Omega}\left|\nabla\left(u_{D}-u_{N}\right)\right|^{2} d x,
$$

and minimizing this functional over a class of admissible domains, where the two state functions $u_{D}$ and $u_{N}$ solve a homogenous Dirichlet $(D)$ and nonhomogenous Neumann $(N)$ problem, respectively:

$$
\begin{aligned}
-\Delta u_{D}=0 & \text { in } \Omega, \\
u_{D}=1 & \text { on } \Gamma, \\
u_{D}=0 & \text { on } \Sigma,
\end{aligned}
$$

and

$$
\begin{aligned}
-\Delta u_{N} & =0 & & \text { in } \Omega, \\
u_{N} & =1 & & \text { on } \Gamma, \\
\frac{\partial u_{N}}{\partial \mathbf{n}} & =\lambda & & \text { on } \Sigma .
\end{aligned}
$$

Bacani and Peichl presented two strategies in computing the first-order shape derivative of the Kohn-Vogelius objective functional. One is by using the Hölder continuity of the two state variables involved [9], and the other one is by using the shape derivatives of states [23]. The authors 
also computed its second-order shape derivative for general domains via the boundary differentiation scheme and via Tiihonen's approach [24]. The computation is found in [25].

In this recent paper, we are going to solve the shape optimization problem using velocity method, wherein we consider nonautonomous velocity fields. The study is important since it confirms a classical result of Delfour and Zolésio in relating shape derivatives of functionals using velocity method and the perturbation of identity technique (cf. [26]).

In the next section (Section 2), we present an overview of some concepts necessary for the understanding of the present study. The section also includes some important results of methods of shape optimization which will be useful in our investigation. We formally present our main contribution which pertains to the form of the second-order shape derivative of the Kohn-Vogelius objective functional in Section 3. Finally, we end our paper by summarizing our results in Section 4.

\section{Preliminaries}

In this section we provide some important (but not exhaustive) background of some shape optimization techniques. We give an overview of these concepts to understand the present study.

2.1. Perturbation of the Identity. Let $\Omega$ and the universal or hold-all domain $U$ be smooth subdomains of $\mathbb{R}^{2}$, such that $\bar{\Omega} \subseteq U$. A class of perturbations $\Omega_{t}$ of the domain $\Omega$ obtained from the perturbation of identity operator $T_{t}$ is defined as

$$
\begin{gathered}
T_{t}=I+t \mathbf{V}: \bar{U} \longrightarrow \mathbb{R}^{2}, \\
T_{t}(x)=x+t \mathbf{V}(x), \quad x \in \bar{U},
\end{gathered}
$$

where the deformation field $\mathbf{V}$ is in $\Theta_{1}$ defined as

$$
\Theta_{1}=\left\{\mathbf{V} \in C^{1,1}\left(\bar{U}, \mathbb{R}^{2}\right):\left.\mathbf{V}\right|_{\Gamma \cup \partial U}=0\right\}
$$

Then, for sufficiently small $t$, (i) $T_{t}: \bar{U} \rightarrow \bar{U}$ is a homeomorphism, (ii) $T_{t}: U \rightarrow U$ is a $C^{1,1}$ diffeomorphism and, in particular, $T_{t}: \Omega \rightarrow \Omega_{t}$ is a $C^{1,1}$ diffeomorphism, (iii) $\Gamma_{t}=T_{t}(\Gamma)=\Gamma$, and $\partial \Omega_{t}=\Gamma \cup \Sigma_{t}$, and (iv) $\partial \Omega_{t}=\Gamma \cup \Sigma_{t}$ (cf. [9, Theorem 7]). For convenience, we will use the following notations throughout the discussion:

$$
\begin{aligned}
I_{t}(x) & =\operatorname{det} D T_{t}(x), \\
M_{t}(x) & =\left(D T_{t}(x)\right)^{-T}, \\
A_{t}(x) & =I_{t}(x) M_{t}^{T}(x) M_{t}(x), \quad x \in \bar{U}, \\
w_{t}(x) & =I_{t}(x)\left|\left(D T_{t}(x)\right)^{-T} \mathbf{n}(x)\right|, \quad x \in \Sigma .
\end{aligned}
$$

The next lemma provides some properties of the transformation $T_{t}$ which are useful in accomplishing our main objective.
Lemma 1 (see $[13,27])$. Consider a fixed vector field $\mathbf{V} \epsilon$ $\Theta_{1}$ and the transformation $T_{t}$ (the perturbation of identity operator). Then, we can find a constant $t_{V}>0$ such that the functions defined above restricted to the interval $I_{V}=\left(-t_{V}, t_{V}\right)$ have the following regularities and properties:

(1) $t \mapsto T_{t} \in C^{1}\left(I_{V}, C^{1}(\bar{U})\right)$,

(2) $t \mapsto I_{t} \in C^{1}\left(I_{V}, C(\bar{\Omega})\right)$,

(3) $t \mapsto w_{t} \in C^{1}\left(I_{V}, C(\bar{\Sigma})\right)$,

(4) $t \mapsto T_{t}^{-1} \in C\left(I_{V}, C^{1}(\bar{U})\right)$,

(5) $t \mapsto A_{t} \in C^{1}\left(I_{V}, C^{1}(\bar{\Omega})\right)$,

(6) $I_{t}=1+t \operatorname{div} \mathbf{V}+t^{2} \operatorname{det} D \mathbf{V}$,

(7) there are positive constants $\alpha_{1}, \alpha_{2}$, and $\beta$ such that $0<$ $\alpha_{1} \leq I_{t}(x) \leq \alpha_{2}$ and $A_{t}(x) \geq \beta I$ for $x \in \Omega$,

(8) $\left.(d / d t) D T_{t}\right|_{t=0}=-\left.(d / d t)\left(D T_{t}\right)^{-1}\right|_{t=0}=D V$,

(9) $\left.(d / d t) T_{t}\right|_{t=0}=\mathbf{V}$,

(10) $\left.(d / d t) I_{t}\right|_{t=0}=\operatorname{div} \mathbf{V}$,

(11) $\lim _{t \downarrow 0} w_{t}=1$,

(12) $\left.(d / d t) A_{t}\right|_{t=0}=A$, where $A=(\operatorname{div} \mathbf{V}) I-\left(D \mathbf{V}+(D \mathbf{V})^{T}\right)$,

(13) $\left.(d / d t) w_{t}\right|_{t=0}=\operatorname{div}_{\Sigma} \mathbf{V}$, where the surface divergence $\operatorname{div}_{\Sigma}$ is defined by

$$
\operatorname{div}_{\Sigma} \mathbf{V}=\left.\operatorname{div} \mathbf{V}\right|_{\Sigma}-(D \mathbf{V n}) \cdot \mathbf{n} .
$$

2.2. The Velocity (Speed) Method (See [5, 20, 21]). In this paper, we are interested in solving the second-order shape derivative of the Kohn-Vogelius objective functional via the velocity method with nonautonomous velocity fields. Detailed discussions about this method can be seen in [5, 20, 21]. We present some of the details here. As our primary interest is focused on nonautonomous velocity fields, we will use the notation $\widehat{\mathbf{V}}$ to denote time-dependent velocity fields in contrast to velocity fields denoted by $\mathbf{V}$ which are not timedependent.

Let $\widehat{\mathbf{V}}:\left[0, t_{V}\right] \times \mathbb{R}^{2} \rightarrow \mathbb{R}^{2}$ be a given velocity field for some fixed $t_{V}>0$. The map $\widehat{\mathbf{V}}$ can be viewed as a family $\{\widehat{\mathbf{V}}(t)\}$ of nonautonomous velocity fields on $\mathbb{R}^{2}$ defined by

$$
x \longmapsto \widehat{\mathbf{V}}(t)(x):=\widehat{\mathbf{V}}(t, x): \mathbb{R}^{2} \longmapsto \mathbb{R}^{2} .
$$

Let $\widehat{\mathbf{V}}$ satisfy

$$
\begin{aligned}
& \text { (V1) } \forall x \in \mathbb{R}^{2}, \widehat{\mathbf{V}}(\cdot, x) \in C\left(\left[0, t_{V}\right) ; \mathbb{R}^{2}\right), \\
& (\mathrm{V} 2) \exists c>0, \forall x, y \in \mathbb{R}^{2},\|\widehat{\mathbf{V}}(\cdot, y)-\widehat{\mathbf{V}}(\cdot, x)\|_{C\left(\left[0, t_{V}\right) ; \mathbb{R}^{2}\right)} \leq \\
& c\|y-x\|,
\end{aligned}
$$

where $\widehat{\mathbf{V}}(\cdot, x)$ denotes the function $t \mapsto \widehat{\mathbf{V}}(t, x)$. Associate with $\widehat{\mathbf{V}}$ the solution $x(t ; X)$ of the ODE:

$$
\frac{d x}{d t}(t)=\widehat{\mathbf{V}}(t, x(t)), \quad t \in[0, \tau], x(0)=X \in \mathbb{R}^{2} .
$$

That is, we suppose that $\widehat{\mathbf{V}}$ is continuous in $t$ and at the same time Lipschitz in spatial variables. We remark that, in the case of autonomous velocity fields, the condition to be satisfied can be simplified as

$$
\left(\mathrm{V}^{\prime}\right) \exists c>0, \forall x, y \in \mathbb{R}^{2},\|\mathbf{V}(x)-\mathbf{V}(y)\| \leq c\|x-y\| .
$$


Remark 2. The above statement can be described equivalently as follows: Let $\widehat{\mathbf{V}}$ belong to $E^{k}=C\left(\left[0, t_{V}\right) ; C^{k}\left(\mathbb{R}^{2}, \mathbb{R}^{2}\right)\right)$ for some integer $k \geq 2$ and a small real number $t_{V}>0$. Let $\Omega \subset$ $\mathbb{R}^{2}$ be a smooth bounded domain with boundary $\Gamma$ which is at least twice differentiable. The field

$$
\widehat{\mathbf{V}}(t)(x)=\widehat{\mathbf{V}}(t, x), \quad x \in \mathbb{R}^{2}
$$

is an element of $C^{k}\left(\mathbb{R}^{2}, \mathbb{R}^{2}\right)$ which may depend on $t \geq 0$. It generates the transformations

$$
T_{t}(\widehat{\mathbf{V}})(X):=T_{t}(X)=x(t ; X), \quad t \geq 0, X \in \mathbb{R}^{2}
$$

through the differential equation

$$
\frac{d x}{d t}(t ; X)=\widehat{\mathbf{V}}(t, x(t ; X)), \quad x(0 ; X)=X .
$$

As already described in the previous section, we denote the transformed domain $T_{t}(\Omega)$ at $t \geq 0$ by $\Omega_{t}$.

Now, the following theorem describes the relation between the perturbation of the identity method and the velocity method. The theorem basically tells us that we can start from either a family of velocity fields $\{\widehat{\mathbf{V}}(t)\}$ on $\mathbb{R}^{N}$ or a family of transformations $\left\{T_{t}\right\}$ of $\mathbb{R}^{N}$ provided that the map $\widehat{\mathbf{V}}, \widehat{\mathbf{V}}(t, x)=\widehat{\mathbf{V}}(t)(x)$, verifies (V1) and (V2) or the map $T, T(t, X)=T_{t}(X)$, verifies assumptions (T1), (T2) and (T3) given below.

Theorem 3 (see [21]). (i) Under assumptions (V1) and (V2) on the map $\widehat{\mathbf{V}}$, the maps $T_{t}$ defined previously have the following properties:

(T1) $\forall X \in \mathbb{R}^{N}, T(\cdot, X) \in C^{1}\left(\left[0, t_{V}\right] ; \mathbb{R}^{N}\right)$, $\exists c>0, \forall X, Y \in \mathbb{R}^{N},\|T(\cdot, Y)-T(\cdot, X)\|_{C^{1}\left(\left[0, t_{V}\right] ; \mathbb{R}^{N}\right)} \leq$ $c|Y-X|$,

(T2) $\forall t \in\left[0, t_{V}\right], X \mapsto T_{t}(X)=T(t, X): \mathbb{R}^{N} \rightarrow \mathbb{R}^{N}$ is bijective,

(T3) $\forall x \in \mathbb{R}^{N}, T^{-1}(\cdot, x) \in C\left(\left[0, t_{V}\right) ; \mathbb{R}^{2}\right)$, $\exists c>0, \forall x, y \in \mathbb{R}^{N},\left\|T^{-1}(\cdot, y)-T^{-1}(\cdot, x)\right\|_{C\left(\left[0, t_{V}\right) ; \mathbb{R}^{2}\right)} \leq$ $c|y-x|$.

(ii) If there exists a real number $t_{V}>0$ and a map $T$ : $\left[0, t_{V}\right] \times \mathbb{R}^{N} \rightarrow \mathbb{R}^{N}$ verifying assumptions (T1), (T2), and (T3), then the map

$$
\begin{aligned}
(t, x) & \longmapsto \widehat{\mathbf{V}}(t, x)=\frac{\partial T}{\partial t}\left(t, T^{-1}(x)\right):\left[0, t_{V}\right] \times \mathbb{R}^{N} \\
& \longrightarrow \mathbb{R}^{N}
\end{aligned}
$$

verifies assumptions (V1) and (V2), where $T_{t}^{-1}$ is the inverse of $X \mapsto T_{t}(X)$.

In the above discussion, we see that the solution to the differential equation (10), $\widehat{\mathbf{V}}=\mathbf{V} \in \Theta_{1}$, is the perturbation of the identity operator $T_{t}$. Conversely, if $T_{t}$ is the perturbation of the identity operator, then (10) is satisfied. Hence, we consider the special case, where $x(t)=I+t \mathbf{V}$, and determine the relationship of the autonomous field $\mathbf{V} \in \Theta_{1}$ and the nonautonomous $\widehat{\mathbf{V}}$ defined in (10). Differentiating $x(t)=$ $I+t \mathbf{V}$ with respect to $t$, we get

$$
\frac{d x}{d t}(t ; X)=\mathbf{V}(X)=\widehat{\mathbf{V}}(t, x(t ; X)), \quad X \in \Omega
$$

This simply implies that $\mathbf{V}(X)=\widehat{\mathbf{V}}(t,(I+t \mathbf{V})(X))$. Now, replacing $X$ by $(I+t \mathbf{V})^{-1}(X)$, we get $\mathbf{V}\left(T_{t}^{-1}(X)\right)=\widehat{\mathbf{V}}(t, X)$ or, equivalently, $\widehat{\mathbf{V}}(t)=\mathbf{V} \circ T_{t}^{-1}$. This is why many results obtained by the perturbation of identity technique can be acquired as well through the velocity method using nonautonomous velocity fields. As an immediate consequence, we note that $\widehat{\mathbf{V}}(0)=\mathbf{V}$. The relation $\widehat{\mathbf{V}}(t)=\mathbf{V} \circ T_{t}^{-1}$ also implies that

$$
\frac{\partial \widehat{\mathbf{V}}}{\partial t}(t)=D \mathbf{V}\left(T_{t}^{-1}\right) \frac{\partial T_{t}^{-1}}{\partial t}
$$

So, in particular, we have

$$
\frac{\partial \widehat{\mathbf{V}}(0)}{\partial t}:=\dot{\hat{\mathbf{V}}}(0)=-[D \mathbf{V}] \mathbf{V}
$$

2.3. Domain and Boundary Transformation. We recall the following theorems on domain and boundary transformations.

Lemma 4 (see [28]). We have the following important transformations which will also be central to our investigation:

(1) Let $\varphi_{t} \in L^{1}\left(\Omega_{t}\right)$. Then $\varphi_{t} \circ T_{t} \in L^{1}(\Omega)$ and

$$
\int_{\Omega_{t}} \varphi_{t} d x_{t}=\int_{\Omega} \varphi_{t} \circ T_{t} I_{t} d x
$$

(2) Let $\varphi_{t} \in L^{1}\left(\Sigma_{t}\right)$. Then $\varphi_{t} \circ T_{t} \in L^{1}(\Sigma)$ and

$$
\int_{\Sigma_{t}} \varphi_{t} d s_{t}=\int_{\Sigma} \varphi_{t} \circ T_{t} w_{t} d s
$$

2.4. Material Derivatives. The material and shape derivatives of the state variables are defined as follows (see $[4,24])$.

Let $u$ be defined in $\left[0, t_{V}\right] \times U$. An element $\dot{u} \in H^{k}(\Omega)$, called the material derivative of $u$, is defined as

$$
\begin{aligned}
\dot{u} & :=\dot{u}(0, x):=\lim _{t \downarrow 0} \frac{u\left(t, T_{t}(x)\right)-u(0, x)}{t} \\
& =\left.\frac{d}{d t} u(t, x+t \mathbf{V}(x))\right|_{t=0}
\end{aligned}
$$

if the limit exists in $H^{k}(\Omega)$.

Remark 5. As pointed out in [9], the material derivative of the state function $u$ can be written as

$$
\dot{u}(x)=\lim _{t \downarrow 0} \frac{u_{t} \circ T_{t}(x)-u(x)}{t}=\left.\frac{d}{d t}\left(u_{t} \circ T_{t}(x)\right)\right|_{t=0}
$$

and it, in fact, characterizes the behavior of the function $u$ at $x \in \Omega \subset U$ in the direction $\mathbf{V}(x)$. 
Now, on the other hand, an element $u^{\prime} \in H^{k}(\Omega)$ is called the shape derivative of $u$ at $\Omega$ at the direction of $\mathbf{V}$, if the following limit exists in $H^{k}(\Omega)$ :

$$
\begin{aligned}
u^{\prime} & :=u^{\prime}(0, x):=\lim _{t \downarrow 0} \frac{u(t, x)-u(0, x)}{t} \\
& :=\left.\frac{\partial}{\partial t} u_{t}(x)\right|_{t=0} .
\end{aligned}
$$

Remark 6. We note that if $\dot{u}$ and $\nabla u \cdot \mathbf{V}$ exist in $H^{k}(\Omega)$, then the shape derivative can be expressed as

$$
u^{\prime}(x)=\dot{u}(x)-(\nabla u \cdot V)(x) .
$$

In general, if $\dot{u}(x)$ and $\nabla u \cdot V(x)$ both exist in $W^{m, p}(\Omega)$, then so does $u^{\prime}(x)$.

2.5. The First- and Second-Order Eulerian Shape Derivatives. We first recall the definition of directional Eulerian shape derivative or simply shape derivative of a shape functional.

Suppose that the shape functional $J: \Omega \rightarrow \mathbb{R}$ is well-defined. Given the deformation field $\widehat{\mathbf{V}}$, the directional Eulerian shape derivative of $J$ at $\Omega$ in the direction of deformation field $\widehat{\mathbf{V}}$ is defined as

$$
d J(\Omega ; \widehat{\mathbf{V}}):=\lim _{t \downarrow 0} \frac{J\left(\Omega_{t}\right)-J(\Omega)}{t}
$$

if the limit exists. The objective functional $J$ is shape differentiable at $\Omega$ provided that $d J(\Omega ; \widehat{\mathbf{V}})$ exists for all $\widehat{\mathbf{V}}$ and if $d J(\Omega ; \widehat{\mathbf{V}})$ is linear and continuous with respect to $\widehat{\mathbf{V}}$. On the other hand, the second-order Eulerian shape derivative of a well-defined shape functional $J$ at $\Omega$ in the direction of the deformation fields $\widehat{\mathbf{V}}$ and $\widehat{\mathbf{W}}$ is defined to be

$$
\begin{aligned}
d^{2} J(\Omega ; \widehat{\mathbf{V}}, \widehat{\mathbf{W}}) & :=\lim _{s \downarrow 0} \frac{d J\left(\Omega_{s}(\widehat{\mathbf{W}}) ; \widehat{\mathbf{V}}\right)-d J(\Omega ; \widehat{\mathbf{V}})}{s} \\
& =\left.\frac{d}{d s}\left(d J\left(\Omega_{s} ; \widehat{\mathbf{V}}\right)\right)\right|_{s=0}
\end{aligned}
$$

if the limit exists. The functional is said to be twice shape differentiable if, for all $\widehat{\mathbf{V}}$ and $\widehat{\mathbf{W}}, d^{2} J(\Omega ; \widehat{\mathbf{V}} ; \widehat{\mathbf{W}})$ exists and if $d^{2} J(\Omega ; \widehat{\mathbf{V}} ; \widehat{\mathbf{W}})$ is bilinear and continuous with respect to $\widehat{\mathbf{V}}$ and $\widehat{\mathbf{W}}$. Following these definitions, the second-order shape derivative of the functional being studied can also be computed as follows:

$$
d^{2} J(\Omega ; \widehat{\mathbf{V}} ; \widehat{\mathbf{W}})=\left.\frac{\partial}{\partial s}\left\{\left.\frac{\partial}{\partial t} d J\left(\Omega_{t, s}\right)\right|_{t=0}\right\}\right|_{s=0} .
$$

In this case, the transported domain $\Omega_{t, s}$ which is a result of two deformation fields $\widehat{\mathbf{V}}$ and $\widehat{\mathbf{W}}$ is illustrated in Figure 3.

Remark 7. Under appropriate hypothesis on the map $\widehat{\mathbf{V}} \mapsto$ $d J(\Omega ; \widehat{\mathbf{V}}(0))$, one can show that $d J(\Omega ; \widehat{\mathbf{V}})=d J(\Omega ; \widehat{\mathbf{V}}(0))$ (cf. [21]). Therefore, if $\widehat{\mathbf{V}}$ is associated with the deformation field $\mathbf{V}$ in the perturbation of the identity, then $d J(\Omega ; \widehat{\mathbf{V}}(0))$

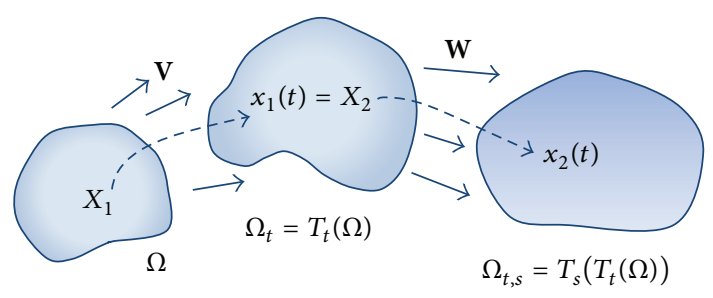

Figure 3: Transport of $\Omega$ after two deformations.

coincides with $d J(\Omega ; \widehat{\mathbf{V}})$ by $\widehat{\mathbf{V}}(0)=\mathbf{V}$. Hence, the firstorder shape derivative of a cost functional obtained via the velocity method coincides with the one obtained from the perturbation of the identity technique.

\section{Analysis for the Nonautonomous Case}

In this section, we will see how important is the expression $\mathbf{V} \circ T_{s}^{-1}$ in considering nonautonomous velocity fields $\widehat{\mathbf{V}}$. Of course if $\mathbf{V}$ is autonomous, $\mathbf{V} \circ T_{s}^{-1}$ is no other than $\widehat{\mathbf{V}}(s)$. Since we are interested in the second-order shape derivative of the Kohn-Vogelius objective functional, we should first recall (without proof) the first-order shape derivative of the KohnVogelius objective functional which is stated in the following theorem.

Theorem 8 (see $[9,22,23])$. For a $C^{1,1}$-bounded domain, the first-order shape derivative of the Kohn-Vogelius (KV) cost functional in the direction of a perturbation field $\widehat{\mathbf{V}} \in \Theta$, where $\widehat{\mathbf{V}} \in \Theta=\left\{\widehat{\mathbf{V}} \in C^{1,1}\left(\bar{U}, \mathbb{R}^{2}\right):\left.\widehat{\mathbf{V}}\right|_{\Gamma \cup \partial U}=0\right\}$ and the state functions $u_{D}$ and $u_{N}$ satisfy the Dirichlet problem $(D)$ and the Neumann problem $(N)$, respectively, is given by

$$
\begin{aligned}
& d J(\Omega ; \widehat{\mathbf{V}})=\frac{1}{2} \\
& \cdot \int_{\Sigma}\left(\lambda^{2}-\left(\nabla u_{N} \cdot \tau\right)^{2}-\left(\nabla u_{D} \cdot \mathbf{n}\right)^{2}+2 \lambda u_{N} \kappa\right) \mathbf{V} \\
& \cdot \mathbf{n} d s .
\end{aligned}
$$

where $\mathbf{n}$ is the unit exterior normal vector to $\Sigma, \tau$ is a unit tangent vector to $\Sigma$, and $\kappa$ is the mean curvature of $\Sigma$.

The above result was first proven by Eppler and Harbrecht in [22]. Other proofs were also given by Bacani and Peichl $[9,23]$, by using two different approaches.

Now, we give our main result of the second-order shape derivative of the Kohn-Vogelius objective functional via velocity method.

Theorem 9. Denote $F=\lambda^{2}-\left(\nabla u_{D} \cdot \mathbf{n}\right)^{2}+2 \lambda \kappa u_{N}-\left(\nabla u_{N} \cdot \tau\right)^{2}$ and let $\widehat{\mathbf{V}}$ and $\widehat{\mathbf{W}}$ be any two velocity fields from the set

$$
\Theta_{2}=\left\{\widehat{\mathbf{V}} \in C^{2,1}\left(\bar{U}, \mathbb{R}^{2}\right):\left.\widehat{\mathbf{V}}\right|_{\text {ГUวU }}=0\right\} .
$$

Assume that, for some sufficiently small $t, d J\left(\Omega_{t}(\widehat{\mathbf{W}}) ; \widehat{\mathbf{V}}(t)\right)$ exists at $\Omega_{t}(\widehat{\mathbf{W}})=T_{t}(\widehat{\mathbf{W}})(\Omega)$ in the direction $\widehat{\mathbf{V}}(t)$. Then, for a $C^{2,1}$-bounded domain, the second-order shape derivative of 
the Kohn-Vogelius (KV) cost functional in the directions of the two perturbation fields $\widehat{\mathbf{V}}$ and $\widehat{\mathbf{W}}$ and the state functions $u_{D}$ and $u_{N}$ which satisfy the Dirichlet problem $(D)$ and the Neumann problem $(N)$, respectively, is given by

$$
\begin{aligned}
& d^{2} J(\Omega ; \widehat{\mathbf{V}} ; \widehat{\mathbf{W}})=\int_{\Sigma} \dot{F}_{W} v_{n}+\left(\frac{\partial F}{\partial \mathbf{n}}+\kappa F\right) v_{n} w_{n} d \Sigma \\
& \quad-\int_{\Sigma} F\left(v_{\Sigma} \cdot D_{\Sigma} \mathbf{n} w_{\Sigma}+\mathbf{n} \cdot D_{\Sigma} \mathbf{v} w_{\Sigma}+\mathbf{n} \cdot D_{\Sigma} \mathbf{w} v_{\Sigma}\right) d \Sigma \\
& \quad+\int_{\Sigma} F(D \mathbf{V}) \mathbf{W} \cdot \mathbf{n}+F \mathbf{V}^{\prime}(0) \cdot \mathbf{n} d \Sigma
\end{aligned}
$$

where $\mathbf{n}$ is the unit exterior normal vector to $\Sigma, \tau$ is a unit tangent vector to $\Sigma$, and $\kappa$ is the mean curvature of $\Sigma$.

Before we proceed in the formal computation of the second-order shape derivative of the Kohn-Vogelius objective functional, we first prove the following auxilliary result.

Lemma 10. Let $F$ and $\dot{F}_{W}$ be, respectively, defined as follows:

$$
\begin{aligned}
F & :=\lambda^{2}-\left(\nabla u_{D} \cdot \mathbf{n}\right)^{2}+2 \lambda \kappa u_{N}-\left(\nabla u_{N} \cdot \tau\right)^{2} \\
\dot{F}_{W} & :=-2\left(\nabla u_{D} \cdot \mathbf{n}\right)\left\{-(D \mathbf{W})^{T} \nabla u_{D} \cdot \mathbf{n}+\nabla u_{D, W}^{\prime} \cdot \mathbf{n}\right. \\
& \left.+\nabla\left(\nabla u_{D} \cdot \mathbf{W}\right) \cdot \mathbf{n}\right\}-2\left(\nabla u_{N} \cdot \tau\right)\left[(-D \mathbf{W})^{T} \nabla u_{N}\right. \\
& \left.+\nabla u_{N, W}^{\prime} \cdot \tau+\nabla\left(\nabla u_{N} \cdot \mathbf{W}\right) \cdot \tau+\nabla u_{N} \cdot \dot{\tau}_{W}\right] \\
& +2 \lambda\left[\dot{\kappa}_{W} u_{N}+\kappa\left(u_{N, W}^{\prime}+\nabla u_{N} \cdot \mathbf{W}\right)\right] .
\end{aligned}
$$

Then, we have

$$
\left.\frac{\partial}{\partial s}\left[\left(F_{s} \circ T_{s}\right) w_{s}\right]\right|_{s=0}=\dot{F}_{W}+F \operatorname{div}_{\Sigma} \mathbf{W} .
$$

Proof. We first note the following:

$$
\begin{aligned}
\left.w_{s}\right|_{s=0} & =1, \\
\left.\left(F_{s} \circ T_{s}\right)\right|_{s=0} & =F \\
\left.\frac{\partial}{\partial s} w_{s}\right|_{s=0} & =\operatorname{div}_{\Sigma} \mathbf{W} .
\end{aligned}
$$

Now, for the expression $\left.(\partial / \partial s)\left(F_{s} \circ T_{s}\right)\right|_{s=0}$, we note that

$$
\begin{aligned}
F_{s} \circ T_{s}= & \left(\lambda^{2} \circ T_{s}\right)-\left(\left(\nabla u_{D, s} \circ T_{s}\right) \cdot \mathbf{n}_{s} \circ T_{s}\right)^{2} \\
& +2\left(\lambda \circ T_{s}\right)\left(\kappa_{s} \circ T_{s}\right)\left(u_{N, s} \circ T_{s}\right) \\
& -\left[\left(\nabla u_{N, s} \circ T_{s}\right) \cdot\left(\tau_{s} \circ T_{s}\right)\right]^{2},
\end{aligned}
$$

and since $\lambda$ is constant, $\lambda \circ T_{s}=\lambda$. Hence, we get

$$
\begin{aligned}
\frac{\partial}{\partial s} & {\left.\left[\left(F_{s} \circ T_{s}\right) w_{s}\right]\right|_{s=0} } \\
& =\left.\left\{\frac{\partial}{\partial s}\left(F_{s} \circ T_{s}\right) w_{s}+\left(F_{s} \circ T_{s}\right) \frac{\partial}{\partial s} w_{s}\right\}\right|_{s=0},
\end{aligned}
$$

by applying product rule. Furthermore, by substitution and distributing the (partial) differential operator, we obtain

$$
\begin{gathered}
\left.\frac{\partial}{\partial s}\left[\left(F_{s} \circ T_{s}\right) w_{s}\right]\right|_{s=0} \\
=\left.\frac{\partial}{\partial s} \lambda^{2}\right|_{s=0}-\left.\frac{\partial}{\partial s}\left[\left[\left(\nabla u_{D, s} \circ T_{s}\right) \cdot\left(\mathbf{n}_{s} \circ T_{s}\right)\right]^{2}\right]\right|_{s=0} \\
\quad+\left.\frac{\partial}{\partial s}\left[2\left(\lambda \circ T_{s}\right)\left(\kappa_{s} \circ T_{s}\right)\left(u_{N, s} \circ T_{s}\right)\right]\right|_{s=0} \\
\quad-\left.\frac{\partial}{\partial s}\left[\left[\left(\nabla u_{N, s} \circ T_{s}\right) \cdot\left(\tau_{s} \circ T_{s}\right)\right]^{2}\right]\right|_{s=0} .
\end{gathered}
$$

Using the chain rule and again the product rule twice, we have

$$
\begin{aligned}
& \left.\frac{\partial}{\partial s}\left[\left(F_{s} \circ T_{s}\right) w_{s}\right]\right|_{s=0}=-2\left(\nabla u_{D} \cdot \mathbf{n}\right) \\
& \left.\quad \cdot \frac{\partial}{\partial s}\left[\left(\nabla u_{D, s} \circ T_{s}\right) \cdot\left(\mathbf{n}_{s} \circ T_{s}\right)\right]\right|_{s=0} \\
& +\left.2 \lambda \frac{\partial}{\partial s}\left(\left(\kappa_{s} \circ T_{s}\right)\left(u_{N, s} \circ T_{s}\right)\right)\right|_{s=0} \\
& \quad-\left.\frac{\partial}{\partial s}\left[\left[\left(\nabla u_{N, s} \circ T_{s}\right) \cdot\left(\tau_{s} \circ T_{s}\right)\right]^{2}\right]\right|_{s=0} \\
& =-2\left(\nabla u_{D} \cdot \mathbf{n}\right) \\
& \quad+\left.\frac{\partial}{\partial s}\left[\left(D T_{s}\right)^{-T} \nabla\left(u_{D, s} \circ T_{s}\right) \cdot\left(\mathbf{n}_{s} \circ T_{s}\right)\right]\right|_{s=0} \\
& \quad+\left.2 \lambda \frac{\partial}{\partial s}\left(\left(\kappa_{s} \circ T_{s}\right)\left(u_{N, s} \circ T_{s}\right)\right)\right|_{s=0}-2\left(\nabla u_{N} \cdot \tau\right) \\
& \quad+\left.\frac{\partial}{\partial s}\left(\left(D T_{s}\right)^{-T} \nabla\left(u_{N, s} \circ T_{s}\right) \cdot\left(\tau_{s} \circ T_{s}\right)\right)\right|_{s=0}=: E_{1} \\
& +E_{2}+E_{3} .
\end{aligned}
$$

Now, we simplify each of the expressions $E_{1}, E_{2}$, and $E_{3}$. In the sequel, we will be needing the material and shape derivatives of the vectors $\mathbf{n}$ and $\tau$ and the mean curvature $\kappa$. For their corresponding forms, we refer the readers to [25, Theorems 4 , 5 , and 6] which are proven in $[5,28]$. So we proceed as follows. First, using the product rule twice and then the chain rule, we get

$$
\begin{aligned}
E_{1} & =-2\left(\nabla u_{D} \cdot \mathbf{n}\right) \\
& \left.\cdot \frac{\partial}{\partial s}\left[\left(D T_{s}\right)^{-T} \nabla\left(u_{D, s} \circ T_{s}\right) \cdot\left(\mathbf{n}_{s} \circ T_{s}\right)\right]\right|_{s=0} \\
& =-2\left(\nabla u_{D} \cdot \mathbf{n}\right)\left\{\left.\frac{\partial}{\partial s}\left[\left(D T_{s}\right)^{-T} \nabla\left(u_{D, s} \circ T_{s}\right)\right]\right|_{s=0}\right. \\
& \left.\left.\cdot\left(\mathbf{n}_{s} \circ T_{s}\right)\right|_{s=0}\right\}-2\left(\nabla u_{D} \cdot \mathbf{n}\right) \\
& \cdot\left\{\left.\left(D T_{s}\right)^{-T} \nabla\left(u_{D, s} \circ T_{s}\right)\right|_{s=0}\right.
\end{aligned}
$$




$$
\begin{aligned}
& \left.\left.\cdot \frac{\partial}{\partial s}\left(\mathbf{n}_{s} \circ T_{s}\right)\right|_{s=0}\right\}=-2\left(\nabla u_{D} \cdot \mathbf{n}\right) \\
& \cdot\left\{\left.\frac{\partial}{\partial s}\left[\left(D T_{s}\right)^{-T} \nabla\left(u_{D, s} \circ T_{s}\right)\right]\right|_{s=0}\right. \\
& \left.\cdot \mathbf{n}+\nabla u_{D} \cdot\left[-\left[(D \mathbf{W})^{T} \mathbf{n}\right]_{\Sigma}\right]\right\}=-2\left(\nabla u_{D} \cdot \mathbf{n}\right) \\
& \cdot\left\{\left.\left.\frac{\partial}{\partial s}\left(D T_{s}\right)^{-T}\right|_{s=0} \nabla\left(u_{D, s} \circ T_{s}\right)\right|_{s=0} \cdot \mathbf{n}\right\} \\
& -2\left(\nabla u_{D} \cdot \mathbf{n}\right)\left\{\left.\left.\left(D T_{s}\right)^{-T}\right|_{s=0} \frac{\partial}{\partial s} \nabla\left(u_{D, s} \circ T_{s}\right)\right|_{s=0}\right. \\
& \cdot \mathbf{n}\}-2\left(\nabla u_{D} \cdot \mathbf{n}\right)\left\{\nabla u_{D} \cdot\left[-\left[(D \mathbf{W})^{T} \mathbf{n}\right]_{\Sigma}\right]\right\} .
\end{aligned}
$$

Interchanging the gradient and the differential operator and upon evaluation of $s$ at 0 , we get

$$
\begin{aligned}
E_{1} & =-2\left(\nabla u_{D} \cdot \mathbf{n}\right) \\
& \cdot\left\{\left[(-D W)^{T} \nabla u_{D}+\nabla\left[\left.\frac{\partial}{\partial s}\left(u_{D, s} \circ T_{s}\right)\right|_{s=0}\right]\right] \cdot \mathbf{n}\right\} \\
& -2\left(\nabla u_{D} \cdot \mathbf{n}\right)\left\{\nabla u_{D} \cdot\left[-\left[(D \mathbf{W})^{T} \mathbf{n}\right]_{\Sigma}\right]\right\} \\
& =-2\left(\nabla u_{D} \cdot \mathbf{n}\right)\left\{-(D \mathbf{W})^{T} \nabla u_{D} \cdot \mathbf{n}+\nabla \dot{u}_{D, W} \cdot \mathbf{n}\right\} \\
& -2\left(\nabla u_{D} \cdot \mathbf{n}\right)\left\{\nabla u_{D} \cdot\left[(D W \mathbf{n} \cdot \mathbf{n}) \mathbf{n}-(D \mathbf{W})^{T} \mathbf{n}\right]\right\} .
\end{aligned}
$$

Here we note that $\dot{u}_{D, W}$ is the material derivative of $u_{D}$ in the direction $\mathbf{W}$. By definition, $\dot{u}_{D, W}$ can be written in terms of the shape derivative $u_{D, W}^{\prime}: \dot{u}_{D, W}=u_{D, W}^{\prime}+\nabla u_{D} \cdot \mathbf{W}$, where $u_{D, W}^{\prime}$ satisfies

$$
\begin{aligned}
-\Delta u_{D, W}^{\prime} & =0 \quad \text { in } \Omega, \\
u_{D, W}^{\prime} & =0 \quad \text { on } \Gamma, \\
u_{D, W}^{\prime} & =-\frac{\partial u_{D}}{\partial \mathbf{n}} \mathbf{W} \cdot \mathbf{n} \quad \text { on } \Sigma .
\end{aligned}
$$

Now, for the second expression $E_{2}$, we have

$$
\begin{aligned}
E_{2} & =\left.2 \lambda \frac{\partial}{\partial s}\left(\left(\kappa_{s} \circ T_{s}\right)\left(u_{N, s} \circ T_{s}\right)\right)\right|_{s=0} \\
& =2 \lambda\left[\left(\frac{\partial}{\partial s}\left(\kappa_{s} \circ T_{s}\right)\right)\left(u_{N, s} \circ T_{s}\right)\right. \\
& \left.+\left(\kappa_{s} \circ T_{s}\right)\left(\frac{\partial}{\partial s}\left(u_{N, s} \circ T_{s}\right)\right)\right]\left.\right|_{s=0}=2 \lambda\left(\dot{\kappa}_{W} u_{N}\right. \\
& \left.+\kappa \dot{u}_{N, W}\right) .
\end{aligned}
$$

Here we stress that $\dot{u}_{N, W}$ is the material derivative of $u_{N}$ in the direction $\mathbf{W}$. Also, $\dot{u}_{N, W}=u_{N, W}^{\prime}+\nabla u_{N} \cdot \mathbf{W}$, where $u_{N, W}^{\prime}$ satisfies

$$
\begin{aligned}
-\Delta u_{N, W}^{\prime} & =0 \quad \text { in } \Omega, \\
u_{N, W}^{\prime} & =0 \quad \text { on } \Gamma, \\
\frac{\partial u_{N, W}^{\prime}}{\partial \mathbf{n}} & =\operatorname{div}_{\Sigma}\left(\mathbf{W} \cdot \mathbf{n} \nabla_{\Sigma} u_{N}\right)+\kappa \lambda \mathbf{W} \cdot \mathbf{n} \quad \text { on } \Sigma .
\end{aligned}
$$

The expression $\dot{\kappa}_{W}$ represents the material derivative of the mean curvature $\kappa$ and it can be shown that $\dot{\kappa}_{W}=$ $-\operatorname{Tr}[D \mathbf{n} D \mathbf{W}]-\left(D\left[(D \mathbf{W n} \cdot \mathbf{n}) \mathbf{n}-(D \mathbf{W})^{T} \mathbf{n}\right]\right) \mathbf{n} \cdot \mathbf{n}$.

Finally, for the last expression $E_{3}$, we have the following:

$$
\begin{aligned}
E_{3}= & -2\left(\nabla u_{N} \cdot \tau\right) \\
& \left.\cdot \frac{\partial}{\partial s}\left(\left(D T_{s}\right)^{-T} \nabla\left(u_{N, s} \circ T_{s}\right) \cdot\left(\tau_{s} \circ T_{s}\right)\right)\right|_{s=0} \\
& =-2\left(\nabla u_{N} \cdot \tau\right) \\
& \left.\cdot\left[\left(\frac{\partial}{\partial s}\left[\left(D T_{s}\right)^{-T} \nabla\left(u_{N, s} \circ T_{s}\right)\right]\right) \cdot\left(\tau_{s} \circ T_{s}\right)\right]\right|_{s=0} \\
& -\left.2\left(\nabla u_{N} \cdot \tau\right)\left[\left(\nabla\left(u_{N, s} \circ T_{s}\right)\right) \cdot \frac{\partial}{\partial s}\left(\tau_{s} \circ T_{s}\right)\right]\right|_{s=0} \\
& =-2\left(\nabla u_{N} \cdot \tau\right) \\
& \cdot\left[\left[(-D W)^{T} \nabla u_{N}+\nabla \dot{u}_{N, W}\right] \cdot \tau+\nabla u_{N} \cdot \dot{\tau}_{W}\right],
\end{aligned}
$$

where $\dot{\tau}_{W}$ is the material derivative of $\tau$ in the direction $\mathbf{W}$ and is given by $\dot{\tau}=\left[(D \mathbf{W})^{T} \mathbf{n} \cdot \tau\right] \mathbf{n}$.

Combining all of these simplification expressions and by the relations in (28), we get the desired result.

Now, we are in the position to prove our main result. In the sequel, we suppose $\widehat{\mathbf{V}}$ and $\widehat{\mathbf{W}}$ to be nonautonomous velocity fields and proceed for the computation as follows. Letting $F_{s}=\lambda^{2}-\left(\nabla u_{D, s} \cdot \mathbf{n}_{s}\right)^{2}+2 \lambda \kappa_{s} u_{N, s}-\left(\nabla u_{N, s} \cdot \tau_{s}\right)^{2}$, we have

$$
\begin{aligned}
d^{2} J & (\Omega ; \widehat{\mathbf{V}} ; \widehat{\mathbf{W}})=\lim _{s \downarrow 0} \frac{d J\left(\Omega_{s}(\widehat{\mathbf{W}}) ; \widehat{\mathbf{V}}\right)-d J(\Omega ; \widehat{\mathbf{V}})}{s} \\
& =\left.\frac{\partial}{\partial s}\left\{\left.\frac{\partial}{\partial t} d J\left(\Omega_{t, s}\right)\right|_{t=0}\right\}\right|_{s=0} \\
& =\frac{\partial}{\partial s}\left(\int_{\Sigma}\left[F_{s}\left(\mathbf{V} \circ T_{s}^{-1}\right) \cdot \mathbf{n}_{s}\right]\right. \\
& \left.\circ T_{s} \operatorname{det} D T_{s}\left|\left(D T_{s}\right)^{-T} \mathbf{n}\right| d \Sigma\right)\left.\right|_{s=0} \\
& =\left.\frac{\partial}{\partial s}\left(\int_{\Sigma}\left[F_{s}\left(\mathbf{V} \circ T_{s}^{-1}\right) \cdot \mathbf{n}_{s}\right] \circ T_{s} w_{s} d \Sigma\right)\right|_{s=0} \\
& =\left.\int_{\Sigma} \frac{\partial}{\partial s}\left\{\left(F_{s} \circ T_{s}\right)\left[\mathbf{V} \cdot\left(\mathbf{n}_{s} \circ T_{s}\right)\right] w_{s}\right\}\right|_{s=0} d \Sigma
\end{aligned}
$$




$$
\begin{aligned}
& =\int_{\Sigma}\left[\left.\frac{\partial}{\partial s}\left(\left(F_{s} \circ T_{s}\right) w_{s}\right)\right|_{s=0}\right][\mathbf{V} \\
& \left.\left.\cdot\left(\mathbf{n}_{s} \circ T_{s}\right)\right|_{s=0}\right] d \Sigma+\int_{\Sigma}\left[\left.\left(F_{s} \circ T_{s}\right) w_{s}\right|_{s=0}\right] \\
& \cdot\left[\left.\frac{\partial}{\partial s}\left\{\mathbf{V} \cdot\left(\mathbf{n}_{s} \circ T_{s}\right)\right\}\right|_{s=0}\right] d \Sigma=: \int_{\Sigma} \Xi_{1} \Xi_{2} d \Sigma \\
& +\int_{\Sigma} \Xi_{3} \Xi_{4} d \Sigma .
\end{aligned}
$$

Note that we already have a simplified form for $\Xi_{1}$ from Lemma 10 , so we only need to simplify the expressions $\Xi_{2}, \Xi_{3}$, and $\Xi_{4}$. For $\Xi_{2}$, we have need for $\Xi_{2}=\left.\mathbf{V} \cdot\left(\mathbf{n}_{s} \circ T_{s}\right)\right|_{s}=\mathbf{V} \cdot \mathbf{n}$ and, for $\Xi_{3}$, we easily get $\Xi_{3}=\left.\left(F_{s} \circ T_{s}\right) w_{s}\right|_{s}=F$, where $F$ is the same with what is given in Lemma 10. Now, for the last expression $\Xi_{4}$, we have

$$
\begin{aligned}
\Xi_{4}= & \left.\frac{\partial}{\partial s}\left\{\mathbf{V} \cdot\left(\mathbf{n}_{s} \circ T_{s}\right)\right\}\right|_{s=0} \\
= & \left.\mathbf{V} \cdot \frac{\partial}{\partial s}\left(\mathbf{n}_{s} \circ T_{s}\right)\right|_{s=0}+\left.\frac{\partial}{\partial s} \mathbf{V}\right|_{s=0} \cdot \mathbf{n} \\
= & \mathbf{V} \cdot\left[(D \mathbf{W n} \cdot \mathbf{n}) \mathbf{n}-(D \mathbf{W})^{T} \mathbf{n}\right]+(D \mathbf{W n} \cdot \mathbf{n}) v_{n} \\
& -(D \mathbf{W}) \mathbf{V} \cdot \mathbf{n}+\mathbf{V}^{\prime}(0) \cdot \mathbf{n}+(D \mathbf{V}) \mathbf{W} \cdot \mathbf{n} .
\end{aligned}
$$

Here, we stress that $\left.(\partial \mathbf{V} / \partial s)\right|_{s=0}=\left.(\partial \mathbf{V}(0) / \partial s)\right|_{s=0}$ and that the relation $\mathbf{V}=\mathbf{V}(s) \circ T_{s}$ implies the identity

$$
\begin{aligned}
\left.\frac{\partial \mathbf{V}}{\partial s}\right|_{s=0} & =\left.\frac{\partial}{\partial s}\left[\mathbf{V}(s) \circ T_{s}\right]\right|_{s=0}=\dot{\mathbf{V}}(0) \\
& =\mathbf{V}^{\prime}(0)+D \mathbf{V W}
\end{aligned}
$$

where $\mathbf{V}^{\prime}(0)(x)=\lim _{t \downarrow 0}(1 / t)[\mathbf{V}(t, x)-\mathbf{V}(0, x)]$.

With these identities, we now have

$$
\begin{aligned}
d^{2} J & (\Omega ; \widehat{\mathbf{V}} ; \widehat{\mathbf{W}})=\int_{\Sigma}\left\{\left[\dot{F}_{W}+F \operatorname{div}_{\Sigma} \mathbf{W}\right] \mathbf{V} \cdot \mathbf{n}\right. \\
& +F\left[(D \mathbf{W n} \cdot \mathbf{n}) v_{n}-(D \mathbf{W}) \mathbf{V} \cdot \mathbf{n}\right] \\
& \left.+\left[\mathbf{V}^{\prime}(0)+(D \mathbf{V}) \mathbf{W}\right] \cdot \mathbf{n}\right\} d \Sigma=\int_{\Sigma}\left\{\dot{F}_{W} v_{n}\right. \\
& +F[\operatorname{div} \mathbf{W}-D \mathbf{W n} \cdot \mathbf{n}] v_{n}+F\left[(D \mathbf{W n} \cdot \mathbf{n}) v_{n}\right] \\
& \left.-F[(D \mathbf{W}) \mathbf{V} \cdot \mathbf{n}]+\left[\mathbf{V}^{\prime}(0)+(D \mathbf{V}) \mathbf{W}\right] \cdot \mathbf{n}\right\} d \Sigma \\
& =\int_{\Sigma}\left\{\dot{F}_{W} v_{n}+F(\operatorname{div} \mathbf{W}) v_{n}-F(D \mathbf{W}) \mathbf{V} \cdot \mathbf{n}\right\} d \Sigma
\end{aligned}
$$

$$
\begin{aligned}
& +\int_{\Sigma}\left\{F(D \mathbf{V}) \mathbf{W} \cdot \mathbf{n}+F \mathbf{V}^{\prime}(0) \cdot \mathbf{n}\right\} d \Sigma \\
& =\int_{\Sigma}\left\{\dot{F}_{W} v_{n}+\left(\frac{\partial F}{\partial \mathbf{n}}+\kappa F\right) v_{n} w_{n}\right\} d \Sigma \\
& -\int_{\Sigma} F\left(v_{\Sigma} \cdot D_{\Sigma} \mathbf{n} w_{\Sigma}+\mathbf{n} \cdot D_{\Sigma} \mathbf{v} w_{\Sigma}+\mathbf{n}\right. \\
& \left.\cdot D_{\Sigma} \mathbf{W} v_{\Sigma}\right) d \Sigma+\int_{\Sigma}\left\{F(D \mathbf{V}) \mathbf{W} \cdot \mathbf{n}+F \mathbf{V}^{\prime}(0)\right. \\
& \cdot \mathbf{n}\} d \Sigma .
\end{aligned}
$$

Now, we note that, for autonomous velocity fields, we have the relation $\widehat{\mathbf{V}}(0)=\mathbf{V}$ (i.e., for $\widehat{\mathbf{V}}(s)=\mathbf{V} \circ T_{s}^{-1}$, we have $\widehat{\mathbf{V}}(0)=$ V). Thus, we now have

$$
\begin{aligned}
& d^{2} J(\Omega ; \mathbf{V}(0) ; \mathbf{W}(0))=d^{2} J(\Omega ; \widehat{\mathbf{V}} ; \widehat{\mathbf{W}})=\int_{\Sigma} \dot{F}_{W} v_{n} \\
& \quad+F(\operatorname{div} \mathbf{W}) v_{n}-F(D \mathbf{W}) \mathbf{V} \cdot \mathbf{n}+F(D \mathbf{V}) \mathbf{W} \cdot \mathbf{n} d \Sigma,
\end{aligned}
$$

from which we see that

$$
\begin{aligned}
d^{2} J(\Omega ; \widehat{\mathbf{V}} ; \widehat{\mathbf{W}})= & d^{2} J(\Omega ; \mathbf{V}(0) ; \mathbf{W}(0)) \\
& +d J\left(\Omega ; \mathbf{V}^{\prime}(0)\right)
\end{aligned}
$$

and here we remark that this result coincides with the one presented by Delfour and Zolésio in [5, page 1420, Equation (28)]. This proves the main Theorem 9.

3.1. On the Boundary Transformation Approach. Now, in this section we compute for the second-order shape derivative of the Kohn-Vogelius objective functional through boundary transformation approach. Again, we assume $\widehat{\mathbf{V}}$ and $\widehat{\mathbf{W}}$ to be nonautonomous velocity fields. So we proceed as follows.

First, using the definition of the second-order shape derivative and by the relation $\widehat{\mathbf{V}}=\mathbf{V} \circ T_{t}^{-1}$, we have

$$
\begin{aligned}
d^{2} J(\Omega ; \widehat{\mathbf{V}} ; \widehat{\mathbf{W}}) & =\left.\frac{\partial}{\partial s}\left\{\left.\frac{\partial}{\partial t} d J\left(\Omega_{t, s}\right)\right|_{t=0}\right\}\right|_{s=0} \\
& =\left.\frac{\partial}{\partial s}\left(\int_{\Sigma_{s}} F_{s} \widehat{\mathbf{V}} \cdot \mathbf{N}_{s} d \Sigma_{s}\right)\right|_{s=0} \\
& =\left.\frac{\partial}{\partial s}\left(\int_{\Sigma_{s}} F_{s}\left(\mathbf{V} \circ T_{s}^{-1}\right) \cdot \mathbf{N}_{s} d \Sigma_{s}\right)\right|_{s=0} .
\end{aligned}
$$

Invoking the boundary differentiation rule, we get

$$
\begin{aligned}
d^{2} J(\Omega ; \widehat{\mathbf{V}} ; \widehat{\mathbf{W}})= & \left.\int_{\Sigma}\left\{\left[\frac{\partial}{\partial \mathbf{n}}\left(F_{s}\left(\mathbf{V} \circ T_{s}^{-1}\right) \cdot \mathbf{N}_{s}\right)+\kappa_{s} F_{s}\left(\mathbf{V} \circ T_{s}^{-1}\right) \cdot \mathbf{N}_{s}\right] \mathbf{W} \cdot \mathbf{n}\right\}\right|_{s=0} d \Sigma \\
& +\left.\int_{\Sigma}\left\{\frac{\partial}{\partial s}\left(F_{s}\left(\mathbf{V} \circ T_{s}^{-1}\right) \cdot \mathbf{N}_{s}\right)\right\}\right|_{s=0} d \Sigma
\end{aligned}
$$




$$
\begin{aligned}
= & \int_{\Sigma}\left\{\left.\left.F_{s}\right|_{s=0} \frac{\partial}{\partial s}\left[\left(\mathbf{V} \circ T_{s}^{-1}\right) \cdot \mathbf{N}_{s}\right]\right|_{s=0}+\left[\frac{\partial}{\partial \mathbf{n}}(F \mathbf{V} \cdot \mathbf{n})+\kappa F \mathbf{V} \cdot \mathbf{n}\right] \mathbf{W} \cdot \mathbf{n}\right\} d \Sigma \\
& +\int_{\Sigma}\left\{\left[\left.\frac{\partial}{\partial s} F_{s}\right|_{s=0}\right]\left[\left.\left(\mathbf{V} \circ T_{s}^{-1}\right) \cdot \mathbf{N}_{s}\right|_{s=0}\right]\right\} d \Sigma \\
= & \int_{\Sigma}\left\{F\left[\left.\left.\frac{\partial}{\partial s}(\mathbf{V}(s))\right|_{s=0} \cdot \mathbf{N}_{s}\right|_{s=0}+\left.\left.\left(\mathbf{V} \circ T_{s}^{-1}\right)\right|_{s=0} \cdot \frac{\partial}{\partial s} \mathbf{N}_{s}\right|_{s=0}\right]\right\} d \Sigma \\
& +\int_{\Sigma}\left\{\left[\frac{\partial}{\partial \mathbf{n}}(F \mathbf{V} \cdot \mathbf{n})+\kappa F \mathbf{V} \cdot \mathbf{n}\right] \mathbf{W} \cdot \mathbf{n}\right\} d \Sigma+\int_{\Sigma} F_{W}^{\prime}(\mathbf{V} \cdot \mathbf{n}) d \Sigma \\
= & \int_{\Sigma}\left\{F\left[(D \mathbf{V})(-\mathbf{W}) \cdot \mathbf{n}+\mathbf{V} \cdot \mathbf{N}^{\prime}\right]+\left[\frac{\partial}{\partial \mathbf{n}}(F \mathbf{V} \cdot \mathbf{n})+\kappa F \mathbf{V} \cdot \mathbf{n}\right] \mathbf{W} \cdot \mathbf{n}+F_{W}^{\prime} v_{n}\right\} d \Sigma \\
= & \int_{\Sigma}\left\{F_{W}^{\prime} v_{n}+F \mathbf{V} \cdot \mathbf{N}^{\prime}+\left[\frac{\partial}{\partial \mathbf{n}}(F \mathbf{V} \cdot \mathbf{n})+\kappa F \mathbf{V} \cdot \mathbf{n}\right] \mathbf{W} \cdot \mathbf{n}+F \dot{\mathbf{V}}(0) \cdot \mathbf{n}\right\} d \Sigma \\
= & \int_{\Sigma}\left\{F_{W}^{\prime} v_{n}+\left(\frac{\partial F}{\partial \mathbf{n}}+\kappa F\right) v_{n} w_{n}-F\left(v_{\Sigma} \cdot D_{\Sigma} \mathbf{n} w_{\Sigma}+\mathbf{n} \cdot D_{\Sigma} \mathbf{V} w_{\Sigma}+\mathbf{n} \cdot D_{\Sigma} \mathbf{W} v_{\Sigma}\right)\right\} d \Sigma \\
& +\int_{\Sigma} F(D \mathbf{V}) \mathbf{W} \cdot \mathbf{n}+F \dot{\mathbf{V}}(0) \cdot \mathbf{n} d \Sigma=d^{2} J(\Omega ; \mathbf{V}(0) ; \mathbf{W}(0))+d J(\Omega ; \dot{\mathbf{V}}(0)) .
\end{aligned}
$$

Remark 11. Here, $d^{2} J(\Omega ; \mathbf{V}(0) ; \mathbf{W}(0))$ is the bilinear term which Delfour and Zolésio refer to as the shape Hessian.

Remark 12. We remark that, as compared to the form of the shape Hessian presented in [22], the result we have established here in Theorem 9 clearly shows the relation pointed in [26] by Delfour and Zolésio about the form of shape Hessians obtained through nonautonomous velocity fields.

\section{Conclusion}

We have computed the second-order shape derivative $d^{2} J(\Omega ; \widehat{\mathbf{V}} ; \widehat{\mathbf{W}})$ of the Kohn-Vogelius objective functional $J_{\mathrm{KV}}$ in the direction of two (nonautonomous) deformation fields $\widehat{\mathbf{V}}$ and $\widehat{\mathbf{W}}$ via velocity method. The computed expression for $d^{2} J(\Omega ; \widehat{\mathbf{V}} ; \widehat{\mathbf{W}})$ is given by

$$
\begin{aligned}
& d^{2} J(\Omega ; \widehat{\mathbf{V}} ; \widehat{\mathbf{W}})=\int_{\Sigma} \dot{F}_{W} v_{n}+\left(\frac{\partial F}{\partial \mathbf{n}}+\kappa F\right) v_{n} w_{n} d \Sigma \\
& \quad-\int_{\Sigma} F\left(v_{\Sigma} \cdot D_{\Sigma} \mathbf{n} w_{\Sigma}+\mathbf{n} \cdot D_{\Sigma} \mathbf{v} w_{\Sigma}+\mathbf{n} \cdot D_{\Sigma} \mathbf{w} v_{\Sigma}\right) d \Sigma \\
& \quad+\int_{\Sigma} F(D \mathbf{V}) \mathbf{W} \cdot \mathbf{n}+F \mathbf{V}^{\prime}(0) \cdot \mathbf{n} d \Sigma
\end{aligned}
$$

where $F=\lambda^{2}-\left(\nabla u_{D} \cdot \mathbf{n}\right)^{2}+2 \lambda \kappa u_{N}-\left(\nabla u_{N} \cdot \tau\right)^{2}, \mathbf{n}$ is the unit exterior normal vector to $\Sigma, \tau$ is a unit tangent vector to $\Sigma$, and $\kappa$ is the mean curvature of $\Sigma$. We stress out that the expression computed for the second-order shape derivative of $J_{\mathrm{KV}}$ is not yet in its explicit form. The explicit form, however, could easily be obtained by substituting all those computed values for each of the expressions found on the integrand or the interested reader may refer directly to [29] for the explicit form of $d^{2} J(\Omega ; \widehat{\mathbf{V}} ; \widehat{\mathbf{W}})$. It is apparent in our first result that the computed expression for the shape derivative coincides with the one presented by Delfour and Zolésio in [5]. Interestingly, the result obtained through boundary transformation approach which again uses nonautonomous velocity fields also verifies the result found by Delfour and Zolésio in [5].

\section{Conflict of Interests}

The authors declare that there is no conflict of interests regarding the publication of this paper.

\section{Acknowledgments}

The authors would like to thank UP Baguio for giving research load credits to write this paper. This work was funded by the UP System Emerging Interdisciplinary Research Program (OVPAA-EIDR-C05-015).

\section{References}

[1] K. K. Choi and N.-H. Kim, Structural Sensitivity Analysis and Optimization 1: Linear Systems, Mechanical Engineering Series, Springer, New York, NY, USA, 2005.

[2] K. Choi and N. Kim, Structural Sensitivity Analysis and Optimization: Nonlinear Systems and Applications, Mechanical Engineering Series, Springer, 2005.

[3] B. Mohammadi and O. Pironneau, Applied Shape Optimization for Fluids, Clardendon Press, Oxford, UK, 2001.

[4] J. Haslinger and R. A. E. Mäkinen, "Introduction to shape optimization: theory, approximation, and computation," in 
Advances in Design and Control, Society for Industrial and Applied Mathematics, 2003.

[5] M. C. Delfour and J.-P. Zolésio, Shapes and Geometries, SIAM, Philadelphia, Pa, USA, 2001.

[6] J. Sokolowski and J.-P. Zolésio, Introduction to Shape Optimization: Shape Sensitivity Analysis, vol. 16 of Springer Series in Computational Mathematics, Springer, 1992.

[7] B. Abda, F. Bouchon, G. Peichl, M. Sayeh, and R. Touzani, "A new formulation for the Bernoulli problem," in Proceedings of the 5th International Conference on Inverse Problems, Control and Shape Optimization, pp. 1-19, Cartagena, Spain, 2010.

[8] H. Kasumba and K. Kunisch, "On computation of the shape Hessian of the cost functional without shape sensitivity of the state variable," SFB-Report 2012-012, 2012.

[9] J. B. Bacani and G. H. Peichl, "On the first-order shape derivative of the Kohn-Vogelius cost functional of the Bernoulli problem," Abstract and Applied Analysis, vol. 2013, Article ID 384320, 19 pages, 2013.

[10] F. Bouchon, S. Clain, and R. Touzani, "Numerical solution of the free boundary Bernoulli problem using a level set formulation," Computer Methods in Applied Mechanics and Engineering, vol. 194, no. 36-38, pp. 3934-3948, 2005.

[11] M. Flucher and M. Rumpf, "Bernoulli's free-boundary problem, qualitative theory and numerical approximation," Journal für die Reine und Angewandte Mathematik, vol. 486, pp. 165-204, 2003.

[12] J. Haslinger, T. Kozubek, K. Kunisch, and G. Peichl, "Shape optimization and fictitious domain approach for solving free boundary problems of Bernoulli type," Computational Optimization and Applications, vol. 26, no. 3, pp. 231-251, 2003.

[13] K. Ito, K. Kunisch, and G. H. Peichl, "Variational approach to shape derivative for a class of Bernoulli problem," Journal of Mathematical Analysis and Applications, vol. 314, no. 1, pp. 126149, 2006.

[14] K. Eppler and H. Harbrecht, "Shape optimization for free boundary problems-analysis and numerics," in Constrained Optimization and Optimal Control for Partial Differential Equations, vol. 160 of International Series of Numerical Mathematics, pp. 277-288, Springer, Basel, Switzerland, 2012.

[15] K. Eppler and H. Harbrecht, "Tracking Neumann data for stationary free boundary problems," SIAM Journal on Control and Optimization, vol. 48, no. 5, pp. 2901-2916, 2009.

[16] F. Murat and J. Simon, "Etude de problemes d'optimal design," in Optimization Techniques Modeling and Optimization in the Service of Man Part 2, vol. 41 of Lecture Notes in Computer Science, pp. 54-62, Springer, Berlin, Germany, 1976.

[17] F. Murat and J. Simon, "Sur le contrôle par un domaine géométrique," Report of L.A. 189 76015, Université Paris VI, 1976.

[18] N. Fujii, "Domain optimization problems with a boundary value problem as a constraint," in Control of Distributed Parameter Systems 1986, pp. 5-9, Pergamon Press, Oxford, UK, 1986.

[19] J. Simon, "Second variations for domain optimization problems," in Control of Distributed Parameter Systems, Birkhäuser, 1988.

[20] M. C. Delfour and J.-P. Zolésio, "Computation of the shape Hessian by a Lagrangian method," in Control of Distributed Parameter Systems, pp. 215-220, IFAC, Perpignan, France, 1989.

[21] M. C. Delfour and J.-P. Zolésio, "Shape Hessian by the velocity method: a Lagrangian approach," in Stabilization of Flexible Structures, vol. 147 of Lecture Notes in Control and Information Sciences, pp. 255-279, Springer, 1990.
[22] K. Eppler and H. Harbrecht, "On a Kohn-Vogelius like formulation of free boundary problems," Computational Optimization and Applications, vol. 52, no. 1, pp. 69-85, 2012.

[23] J. B. Bacani and G. H. Peichl, "Solving the exterior Bernoulli problem using the shape derivative approach," in Mathematics and Computing 2013, R. N. Mohapatra, D. Giri, P. K. Saxena, and P. D. Srivastava, Eds., vol. 91 of Springer Proceedings in Mathematics \& Statistics, pp. 251-269, Springer, New Delhi, India, 2014.

[24] T. Tiihonen, "Shape optimization and trial methods for free boundary problems," RAIRO: Modélisation Mathématique et Analyse Numérique, vol. 31, no. 7, pp. 805-825, 1997.

[25] J. B. Bacani and G. H. Peichl, “The second-order shape derivative of kohn-vogelius-type cost functional using the boundary differentiation approach," Mathematics, vol. 2, no. 4, pp. 196-217, 2014.

[26] M. C. Delfour and J.-P. Zolésio, "Velocity method and Lagrangian formulation for the computation of the shape Hessian," SIAM Journal on Control and Optimization, vol. 29, no. 6, pp. 1414-1442, 1991.

[27] J. Haslinger, K. Ito, T. Kozubek, K. Kunisch, and G. Peichl, “On the shape derivative for problems of Bernoulli type," Interfaces and Free Boundaries, vol. 11, no. 2, pp. 317-330, 2009.

[28] J. Sokolowski and J.-P. Zolésio, Introduction to Shape Optimization, Springer, Berlin, Germany, 1991.

[29] J. B. Bacani, Methods of shape optimization in free boundary problems [Ph.D. thesis], Karl-Franzens-Universität Graz, Graz, Austria, 2013. 


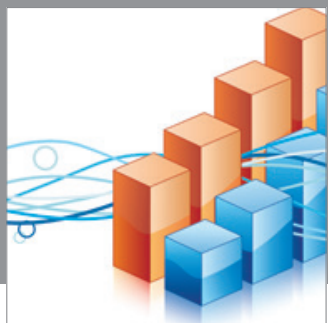

Advances in

Operations Research

mansans

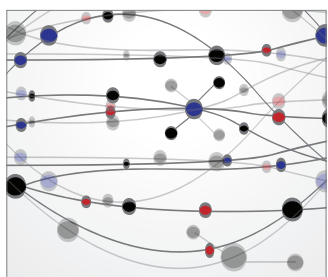

The Scientific World Journal
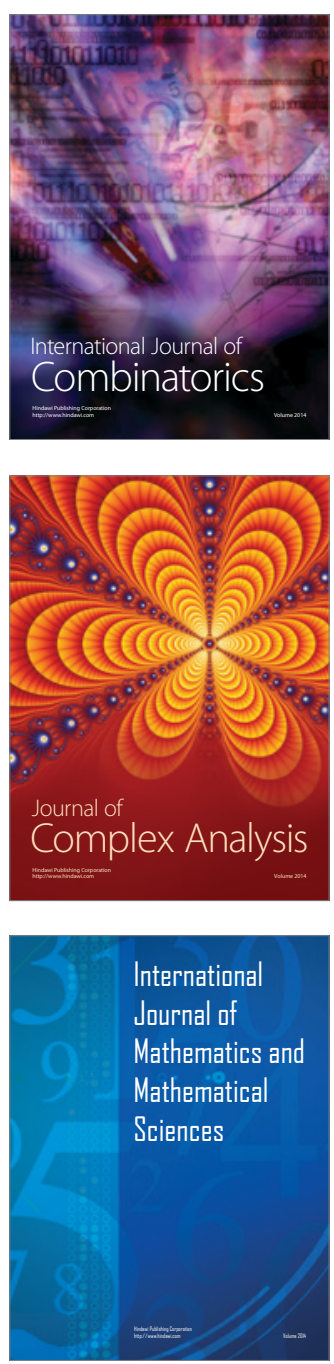
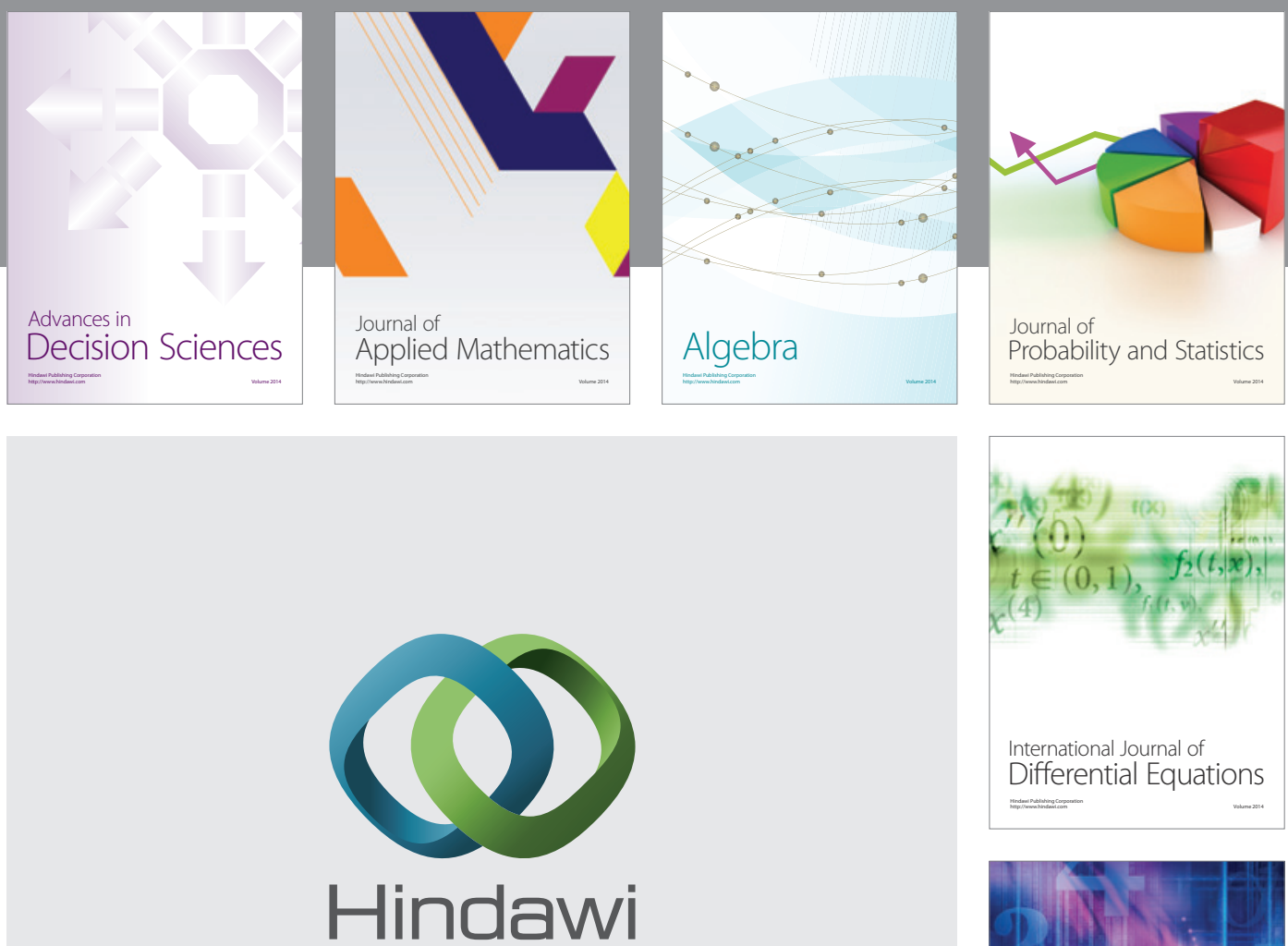

Submit your manuscripts at http://www.hindawi.com
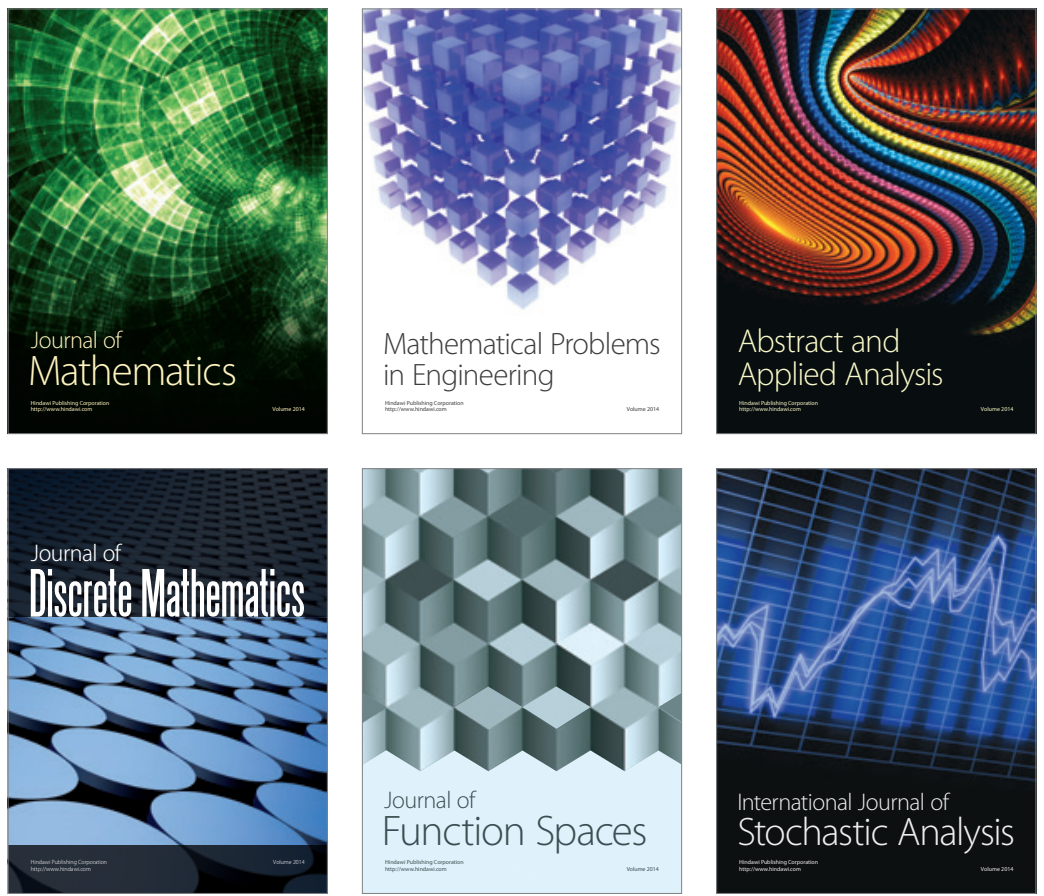

Journal of

Function Spaces

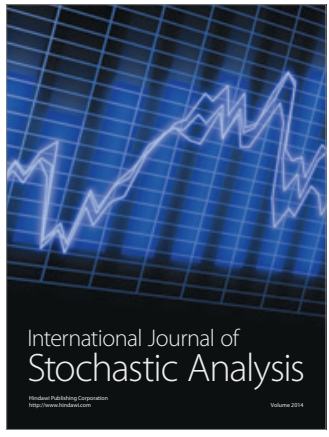

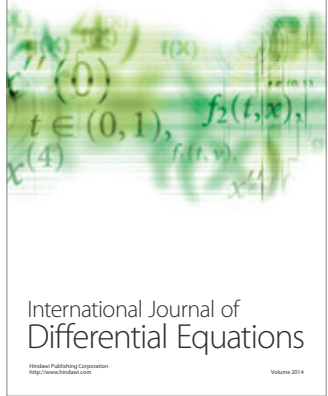
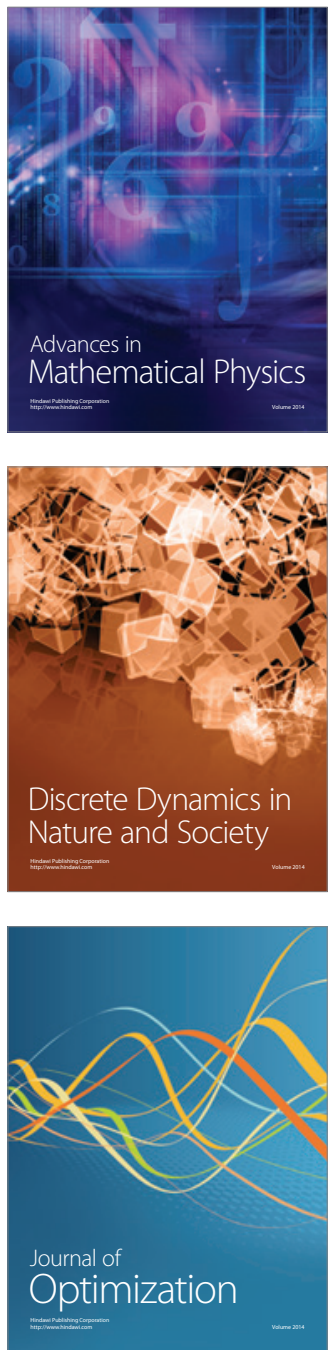